EPJ Web of Conferences 19, 05013 (2012)

DOI: $10.1051 /$ epjconf/20121905013

(C) Owned by the authors, published by EDP Sciences, 2012

\title{
Spectral diagnostics of late-type stars: Non-LTE and <3D> approach
}

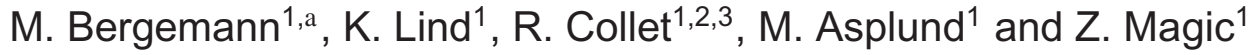 \\ ${ }^{1}$ Max-Planck-Institute for Astrophysics, Karl-Schwarzschild Str. 1, Garching, Germany \\ 2 Centre for Star and Planet Formation, Natural History Museum of Denmark University of \\ Copenhagen, OEster Voldgade 5-7, 1350 Copenhagen, Denmark \\ 3 Astronomical Observatory/Niels Bohr Institute, Juliane Maries Vej 30, \\ 2100 Copenhagen, Denmark
}

\begin{abstract}
We determine effective temperature, metallicity, and microturbulence for a number of wellstudied late-type stars. We use the new NLTE atomic model of Fe, and discuss the results for the MARCS models, as well as for the spatial and temporal averages of full 3D hydrodynamical simulations of stellar convection. It is shown that, contrary to the mean 3D models, certain limitations shall be imposed on the line formation and spectrum synthesis calculations with classical hydrostatic 1D models to obtain physicallyrealistic results.
\end{abstract}

\section{INTRODUCTION}

In the past decade, there has been a dramatic increase in our understanding of Galactic chemical evolution, primarily through the rapidly growing amount of observational data. Largescale spectroscopic surveys, such as the on-going SDSS/SEGUE and the forth-coming Gaia-ESO programmes, aim to obtain millions of spectra that will allow to uniquely characterize various stellar populations based on their chemical properties. The automated pipelines designed for the analysis of these huge observational archives shall be fast and efficient, therefore a number of simplifying physical assumptions are usually introduced. Stellar atmospheres are approximated by 1D plane-parallel or spherically-symmetric systems, which are in hydrostatic and local thermodynamic (LTE) equilibrium.

Here, we discuss how these approximations affect the determination of stellar parameters from spectra of FGK stars. We compute effective temperature and metallicity for a number of reference stars relaxing the assumptions of LTE and 1D hydrostatic equilibrium. The details of the study will be described elsewhere (Bergemann et al. in prep, Lind et al. in prep.)

\section{SPECTROSCOPIC STELLAR PARAMETERS}

We constructed a new non-LTE (NLTE) model atom of Fe using all theoretical and experimental atomic data available so far. The model has been applied to the analysis of the Sun and a number of well-studied late-type stars with parameters determined by other independent methods, including the metal-poor turnoff star HD 84937 and the giant HD 122563. Calculations of the NLTE spectral line formation for Fe were performed with the flux-constant 1D LTE MARCS model atmospheres (Gustafsson et al. 2008), as well as spatial and temporal averages of the full 3D hydrodynamical simulations of stellar convection computed with the Stagger code (Nordlund \& Galsgaard 1995, see also Collet et al. 2011).

\footnotetext{
a e-mail: mbergema@mpa-garching.mpg.de
}

This is an Open Access article distributed under the terms of the Creative Commons Attribution-Noncommercial License 3.0, which permits unrestricted use, distribution, and reproduction in any noncommercial medium, provided the original work is properly cited. 

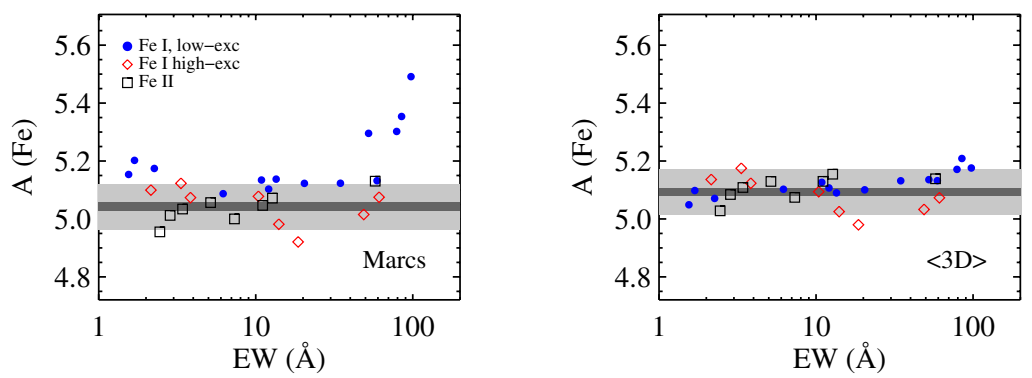

Figure 1. NLTE abundances for HD 140283 obtained with the MARCS (left) and mean 3D model atmospheres using the same procedure and the linelist (see text).

For the abundance calculations, we used high-quality spectra from the UVESPOP database (Bagnulo et al. 2003) and typically 20-60 Fe I and Fe II lines with the lower level excitation potential of $0-5 \mathrm{eV}$ were analysed per star. Surface gravities were determined from the Hipparcos parallaxes (van Leeuwen 2007) and effective temperatures were taken from the IRFM study of Casagrande et al. (2010). The efficiency of inelastic collisions with $\mathrm{H}$ I atoms was constrained based on the excitation-ionization equilibrium of $\mathrm{Fe} \mathrm{I} / \mathrm{Fe}$ II allowing for a small variation of $T_{\text {eff }}$ within its methodic error. Microturbulence was also found iteratively. The results for one of the reference stars, a metal-poor subgiant HD 140283, are shown in Fig. 1.

We find that the excitation-ionization equilibrium of $\mathrm{Fe}$ in all reference stars can be well established under NLTE with the mean 3D model atmospheres. Analysis with the MARCS models delivers consistent abundances from the lines of both ionization stages, but only if the Fe I lines with high excitation potential are used; low-excitation lines give discrepant results pointing at systematic errors introduced by the assumptions of $1 \mathrm{D}$ geometry and hydrostatic equilibrium in the $1 \mathrm{D}$ models. It is, thus, recommended to exclude resonance and subordinate $\mathrm{Fe} I$ lines in 1D spectroscopic studies to avoid systematic bias in metallicity. The $[\mathrm{Fe} / \mathrm{H}]_{1 \mathrm{D}}$ and $[\mathrm{Fe} / \mathrm{H}]_{<3 \mathrm{D}>}$ agree only as long as the high-excitation Fe I lines are employed.

\section{CONCLUSIONS}

We perform a spectroscopic analysis of a small number of late-type stars with parameters determined by other independent methods. The ionization and excitation balance of Fe I/Fe II can be achieved with the mean 3D model atmospheres under NLTE. Strong resonance and subordinate Fe I lines are sensitive to the $T(\tau)$ relation of the model atmospheres, thus, classical 1D models fail to provide consistent excitation balance. The NLTE spectroscopic effective temperatures and metallicities, inferred for the standard stars, agree well in 1D and $<3 \mathrm{D}>$ only if high-excitation Fe I lines are used.

\section{References}

[1] B. Gustafsson, B. Edvardsson, K. Eriksson, et al., A\&A 486, (2008) 951-970

[2] Å. Nordlund, R. F. Stein, and M. Asplund, Living Reviews in Solar Physics 6, (2009)

[3] R. Collet, Z. Magic, and M. Asplund, Proceedings of the GREAT-ESF Workshop: "Stellar Atmospheres in the Gaia Era", (2011)

[4] H.-W. Drawin, Zeitschrift für Physik 225, (1969) 483

[5] S. Bagnulo, E. Jehin, C. Ledoux, et al., ESO Messenger 114, (2003) 10-14

[6] F. Van Leeuwen, A\&A 474, (2007) 653-664

[7] L. Casagrande, I. Ramírez, J. Melélendez, M. Bessell, M. Asplund, A\&A 512, (2010) 